\title{
Editorial: Welcoming Hugues Chaté to Physical Review Letters
}

My appointment as APS Editor in Chief last August created an opening in the Physical Review Letters (PRL) Lead Editor position. Dr. Hugues Chaté agreed to take the position on an interim basis and has done a truly outstanding job in that capacity. In the past six months he has developed close working relationships with the PRL editorial staff, authors, referees, and readers, as well as with the editorial teams of the full APS family of journals. Based on this, and on additional input that consistently confirms my high opinion of Hugues, I asked him to serve as the permanent PRL Lead Editor. It is my great pleasure to announce that he agreed.

Hugues's research covers a wide spectrum of topics ranging from nonlinear dynamics to statistical physics and critical phenomena to active matter. He enjoys a superb and welldeserved international reputation, and serves as an outstanding APS and PRL ambassador both in Europe, where he is a Senior Scientist at the Service of Condensed Matter Physics of the French Commissariat à l'Energie Atomique (CEA), as well as on the Pacific Rim and in China, where he holds a part-time faculty position at the Beijing Computational Science Research Center. Hugues's international experience also includes an earlier postdoctoral stay at Bell Laboratories, and service as the leader of the Advanced Study Group "Statistical Physics of Collective Motion" at the Max Planck Institute for the Physics of Complex Systems in Dresden, Germany. His previous editorial experience includes service on the editorial board of Physical Review $E$ and as a Divisional Associate Editor of PRL.

I am certain that PRL will benefit greatly from Hugues's leadership, energy, and broad vision. Please join me in welcoming Hugues Chaté to his very important new role in the APS family of journals.

Published 4 April 2017

Pierre Meystre

Editor in Chief

American Physical Society

DOI: 10.1103/PhysRevLett.118.140001 